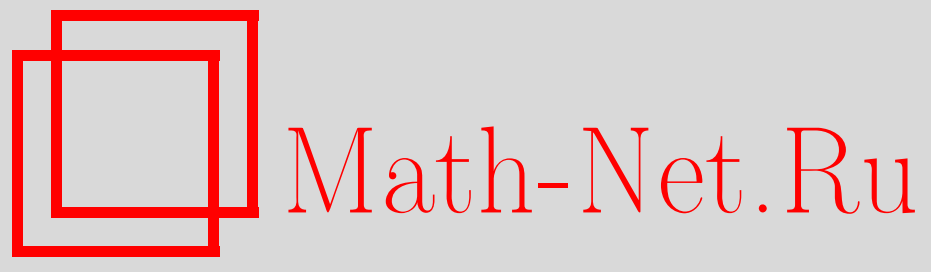

Ю. С. Коломойцев, Мультипликативные достаточные условия для мультипликаторов Фурье, Изв. РАН. Сер. матем., 2014, том 78, выпуск 2, 145-166

DOI: https://doi.org/10.4213/im8078

Использование Общероссийского математического портала Math-Net.Ru подразумевает, что вы прочитали и согласны с пользовательским соглашением http://www . mathnet.ru/rus/agreement

Параметры загрузки:

IP : 34.229 .108 .108

26 апреля 2023 г., 13:37:20

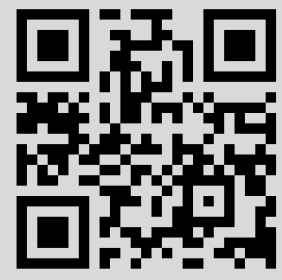


УДК 517.5

\author{
Ю. С. Коломойцев
}

\title{
Мультипликативные достаточные условия для мультипликаторов Фурье
}

\begin{abstract}
Получены новые достаточные условия для мультипликаторов Фурье в пространствах Харди $H_{p}\left(\mathbb{R}^{n}\right), 0<p \leqslant 1$, и $L_{p}\left(\mathbb{R}^{n}\right), 1 \leqslant p \leqslant \infty$. Эти условия имеют мультипликативный характер и даются в терминах совместного поведения "норм" функций в $L_{q}\left(\mathbb{R}^{n}\right)$ и пространствах Бесова $B_{r, \infty}^{s}\left(\mathbb{R}^{n}\right)$.

Библиография: 27 наименований.
\end{abstract}

Ключевые слова: мультипликаторы Фурье, пространства Харди, пространства Бесова, алгебра Винера.

DOI: $10.4213 / \operatorname{im} 8078$

\section{$\S 1$. Введение}

С. Г. Михлин в 1956 г. получил [1] следующий известный результат о мультипликаторах Фурье.

ТЕОРемА А. Если для ограниченной функции $m$ выполняется неравенство

$$
\left|D^{\nu} m(\xi)\right| \leqslant C|\xi|^{-|\nu|_{1}}, \quad \xi \in \mathbb{R}^{n} \backslash\{0\},
$$

для всех $|\nu|_{1} \leqslant[n / 2]+1$ (әде [·] - целая часть числа), то функиия $m$ является мультипликатором Фуръе в $L_{p}\left(\mathbb{R}^{n}\right)$ при всех $1<p<\infty$.

Позже Л. Хёрмандер [2] показал, что данный результат останется верным, если для функции $m$ потребовать более слабое условие вида

$$
\left(R^{-n} \int_{R<|\xi|<2 R}\left|D^{\nu} m(\xi)\right|^{2} d \xi\right)^{\frac{1}{2}} \leqslant C R^{-|\nu|_{1}}, \quad 0<R<\infty
$$

которое также должно выполняться для всех $|\nu|_{1} \leqslant[n / 2]+1$.

Эти результаты усиливали и обобщали многие известные математики. Укажем здесь лишь некоторые работы, имеющие отношение к настоящей статье, а именно [3]--[11]. В частности, в [3], [4], [6], [11] было показано, что в (1) вместо частных производных целого порядка можно брать дробные степени оператора Бесселя, а вместо $L_{2}$-нормы $-L_{q}$-норму для $1<q<\infty$. При этом в зависимости от $q$ возрастает или убывает количество производных, для которых данное условие следует проверять. Отметим также, что в [5] и [6] был получен аналог результата Л. Хёрмандера в вещественных пространствах Харди $H_{p}\left(\mathbb{R}^{n}\right)$, $0<p \leqslant 1$.

Дальнейшие усиления достаточных условий С. Г. Михлина и Л. Хёрмандера связаны с пространствами Бесова $B_{p, q}^{s}\left(\mathbb{R}^{n}\right)$ (см., например, [4], [9]-[11]).

(C) Ю. С. Коломойцев, 2014 
Пусть $\varphi$ - некоторая гладкая функция, имеющая компактный носитель и образующая разбиение единицы (см. далее (7)). В работах [4] и [11] было показано, что функция $m$ является мультипликатором Фурье в $L_{p}\left(\mathbb{R}^{n}\right)$ при любом $1<p<\infty$, если

$$
\sup _{\delta>0}\|m(\delta \cdot) \varphi\|_{B_{2, \lambda}^{s}}<\infty
$$

где $s>n / 2$ и $\lambda=1$. Более того, в [10] и [11] был рассмотрен предельный случай данного утверждения, т. е. $s=n / 2$, однако при дополнительных ограничениях, налагаемых на функцию $m$, или при некотором другом определении нормы в $B_{2,1}^{n / 2}\left(\mathbb{R}^{n}\right)$.

Для мультипликаторов в пространствах $H_{p}\left(\mathbb{R}^{n}\right), 0<p<1$, были получены более точные результаты. Так, в работе [9] показано, что функция $m$ является мультипликатором Фурье в $H_{p}\left(\mathbb{R}^{n}\right), 0<p<1$, если $(2)$ выполняется при $s=$ $n(1 / p-1 / 2)$ и $\lambda=p$ (см. также [12] для случая $p=1)$.

А. Миячи в работах [7] и [8] получил достаточные условия иного типа, учитывающие совместное поведение функции и ее частных производных и, таким образом, позволяющие достаточно эффективно исследовать важные функциимультипликаторы вида

$$
m_{\alpha, \beta}(\xi)=\rho(\xi)|\xi|^{-\beta} e^{i|\xi|^{\alpha}}
$$

Такие функции имеют тесную связь с задачей Коши для уравнения Шрёдингера и волнового уравнения.

В частности, в [7] был получен следующий результат.

Теорема В. Пусть $0<p<2, a, b \geqslant 0 u k=\left[n\left(\frac{1}{\min (p, 1)}-\frac{1}{2}\right)\right]+1$. Предположим, что $m \in W_{2, \text { lос }}^{k}\left(\mathbb{R}^{n}\right), m(\xi)=0$ в окрестности нуля и $m(\xi)$ удовлетворяет неравенствам

$$
|m(\xi)| \leqslant C|\xi|^{-b}, \quad\left(R^{-n} \int_{R<|\xi|<2 R}\left|D^{\nu} m(\xi)\right|^{2} d \xi\right)^{\frac{1}{2}} \leqslant C R^{-b} R^{(a-1)|\nu|_{1}}
$$

для всех $|\nu|_{1} \leqslant k$ u $R>0$. Тогда если $b \geqslant a n(1 / p-1 / 2)$, то $m$ является мультипликатором Фуръе в $H_{p}\left(\mathbb{R}^{n}\right)$. Если $0<p<1$, то первое неравенство в (4) можно не учитыватъ.

Аналогичный результат имеет место и для функций $m$, имеющих компактный носитель (см. [7, теоремы $\left.\left.1^{\prime}, 1^{\prime \prime}, 2^{\prime \prime}\right]\right)$.

Отметим, что подобные достаточные условия для мультипликаторов степенных рядов в аналитических пространствах Харди $H_{p}\left(D^{n}\right), 0<p \leqslant 1$, были получены в [13].

В [14] было показано, что если функция $m$ вместо неравенств (4) удовлетворяет более слабому условию

$$
\sup _{\delta>0}\|m(\delta \cdot) \varphi\|_{L_{2}}^{1-\theta}\|m(\delta \cdot) \varphi\|_{W_{2}^{k}}^{\theta}<\infty, \quad \theta=\frac{n}{k}\left(\frac{1}{p}-\frac{1}{2}\right),
$$

то она будет мультипликатором в $H_{p}\left(\mathbb{R}^{n}\right), 0<p<1$, а также и в $H_{p}\left(\mathbb{R}^{n}\right)$ при $1 \leqslant p<2$, если вместо $L_{2}$-нормы в $(5)$ поставить $L_{\infty}$-норму. 
Условия вида (5) естественно называть мультипликативными достаточнъми условиями. Отличительная черта таких условий заключается в том, что производные функции $m$ могут вести себя в определенном смысле довольно "плохо", например иметь экспоненциальный рост на бесконечности или в окрестности нуля. Однако если функция $m$ ведет себя "хорошо" на участках "плохого" поведения своих производных, то при определенных условиях такая функция является мультипликатором Фурье.

Отметим, что для мультипликаторов Фурье в пространстве $L_{1}\left(\mathbb{R}^{n}\right)$ (или $\left.L_{\infty}\right)$ достаточные условия в терминах совместного поведения функции и ее производных появились совсем недавно (см., например, работы [15], [16] и обзор [17, $\S 10])$. В частности, в [16] было показано, что если $m \in C(\mathbb{R}), \lim m(\xi)=0$ при $|\xi| \rightarrow \infty, m$ локально абсолютно непрерывна на $\mathbb{R}$ и, дополнительно, при $1<p, q<\infty$ и $1 / p+1 / q>1$ выполнено

$$
m \in L_{p}(\mathbb{R}), \quad m^{\prime} \in L_{q}(\mathbb{R}),
$$

то $m$ является мультипликатором Фурье в $L_{1}(\mathbb{R})$.

В настоящей работе получены новые мультипликативные достаточные условия вида (5), которые сочетают в себе условия вида (2) и (6). В частности, показано, что в (5) вместо нормы в пространствах Соболева $W_{2}^{k}$ можно взять норму в пространствах Бесова $B_{r, \infty}^{s}$, а вместо $L_{2}$-нормы $-L_{q}$-норму, при этом установлена точная связь между $n, p, q, r$ и $s$. Аналогичные результаты получены для мультипликаторов в пространстве $L_{1}\left(\mathbb{R}^{n}\right)$.

Настоящая статья имеет следующую структуру. В $§ 2$ даются необходимые определения и обозначения. В $\S 3$ формулируются основные результаты, а также предложения, показывающие их точность. В $\S 4$ формулируются некоторые следствия и замечания. В $\S 5$ приводятся необходимые свойства пространств Бесова, а также некоторые вспомогательные леммы. В $\S 6$ доказываются результаты статьи.

\section{§ 2. Основные обозначения}

Пусть $\mathbb{R}^{n}-n$-мерное евклидово пространство элементов $x=\left(x_{1}, \ldots, x_{n}\right)$, $\xi=\left(\xi_{1}, \ldots, \xi_{n}\right)$ со скалярным произведением $(x, \xi)=x_{1} \xi_{1}+\cdots+x_{n} \xi_{n}$ и нормой $|x|=(x, x)^{1 / 2}$. Как обычно, пространство $L_{p}\left(\mathbb{R}^{n}\right)$ состоит из измеримых функций $f(x), x \in \mathbb{R}^{n}$, для которых при $0<p<\infty$

$$
\|f\|_{p}=\|f\|_{L_{p}}=\left(\int_{\mathbb{R}^{n}}|f(x)|^{p} d x\right)^{\frac{1}{p}}<\infty,
$$

а при $p=\infty$

$$
\|f\|_{\infty}=\|f\|_{L_{\infty}}=\operatorname{esssup}_{x \in \mathbb{R}^{n}}|f(x)|<\infty .
$$

Через $C\left(\mathbb{R}^{n}\right)$ обозначим пространство всех ограниченных равномерно непрерывных на $\mathbb{R}^{n}$ функций. При $k \in \mathbb{N} \cup\{0\}$ положим

$$
C^{k}\left(\mathbb{R}^{n}\right)=\left\{f: D^{\nu} f \in C\left(\mathbb{R}^{n}\right) \quad \forall|\nu|_{1} \leqslant k\right\} .
$$


При этом

$$
\|f\|_{C^{k}}=\sum_{|\nu|_{1} \leqslant k}\left\|D^{\nu} f\right\|_{\infty} .
$$

Здесь и далее $\nu-n$-мерный вектор с неотрицательными целыми координатами, $|\nu|_{1}=\nu_{1}+\cdots+\nu_{n}$ и

$$
D^{\nu} f=\frac{\partial^{|\nu|_{1}} f}{\partial x_{1}^{\nu_{1}} \ldots \partial x_{n}^{\nu_{n}}}, \quad D^{0} f=f .
$$

Далее также понадобится следующий класс функций:

$$
C_{0}\left(\mathbb{R}^{n}\right)=\left\{f: f \in C\left(\mathbb{R}^{n}\right), \lim _{|x| \rightarrow \infty} f(x)=0\right\} .
$$

Мы будем использовать стандартные обозначения для пространства распределений умеренного роста $\mathscr{S}^{\prime}\left(\mathbb{R}^{n}\right)$ и для соответствующего пространства пробных функций $\mathscr{S}\left(\mathbb{R}^{n}\right)$.

Вещественные пространства Харди $H_{p}\left(\mathbb{R}^{n}\right), 0<p<\infty$, определяют класс умеренных распределений $f \in \mathscr{S}^{\prime}\left(\mathbb{R}^{n}\right)$ таких, что

$$
\|f\|_{H_{p}}=\left\|\sup _{t>0}\left|\varphi_{t} * f\right|\right\|_{p}<\infty
$$

где $\varphi \in \mathscr{S}\left(\mathbb{R}^{n}\right), \int_{\mathbb{R}^{n}} \varphi(x) d x \neq 0$ и $\varphi_{t}(x)=t^{-n} \varphi(x / t)$.

Хорошо известно, что если $1<p<\infty$, то $H_{p}\left(\mathbb{R}^{n}\right)=L_{p}\left(\mathbb{R}^{n}\right)$ с эквивалентными нормами (см. [5]).

Преобразование Фурье функции $f \in L_{1}\left(\mathbb{R}^{n}\right)$ определим стандартным обра30м:

$$
\widehat{f}(\xi)=\mathscr{F} f(\xi)=(2 \pi)^{-\frac{n}{2}} \int_{\mathbb{R}^{n}} f(x) e^{-i(\xi, x)} d x,
$$

положим также $\mathscr{F}^{-1} f(\xi)=\mathscr{F} f(-\xi)$. Далее операторы $\mathscr{F}$ и $\mathscr{F}^{-1}$ будем понимать в обобщенном смысле.

Функцию $m$ называют мультипликатором Фуръе в $H_{p}\left(\mathbb{R}^{n}\right)$ (будем записывать $\left.m \in \mathscr{M}\left(H_{p}\right)\right)$, если оператор

$$
T: \mathscr{F}(T f)(\xi)=m(\xi) \widehat{f}(\xi), \quad f \in H_{p}\left(\mathbb{R}^{n}\right) \cap L_{2}\left(\mathbb{R}^{n}\right),
$$

можно продолжить до линейного ограниченного оператора на всем $H_{p}\left(\mathbb{R}^{n}\right)$. При этом

$$
\|T\|_{H_{p} \mapsto H_{p}}=\|m\|_{\mathscr{M}\left(H_{p}\right)}=\sup _{\|f\|_{H_{p}} \leqslant 1}\|T f\|_{H_{p}} .
$$

Аналогичным образом определяются мультипликаторы Фурье в пространстве $L_{1}\left(\mathbb{R}^{n}\right)$ (или $\left.L_{\infty}\right)$.

Напомним некоторые элементарные свойства мультипликаторов Фурье (см., например, [18, с. 113, 114] или [19, гл. 6]):

(i) если $1<p<2$, то $\mathscr{M}\left(L_{1}\right) \subset \mathscr{M}\left(L_{p}\right) \subset \mathscr{M}\left(L_{2}\right)=L_{\infty}\left(\mathbb{R}^{n}\right)$;

(ii) если $1 \leqslant p \leqslant \infty$ и $1 / p+1 / p^{\prime}=1$, то $\mathscr{M}\left(L_{p}\right)=\mathscr{M}\left(L_{p^{\prime}}\right)$;

(iii) если $m_{1}, m_{2} \in \mathscr{M}\left(H_{p}\right)$, то $m_{1}+m_{2} \in \mathscr{M}\left(H_{p}\right)$ и $m_{1} m_{2} \in \mathscr{M}\left(H_{p}\right)$. 
Согласно свойству (ii) для исследования мультипликаторов Фурье в пространствах $L_{p}\left(\mathbb{R}^{n}\right), 1<p<\infty$, достаточно рассматривать только случай $1<p<2$.

Формулировки основных теорем настоящей работы даны в терминах "норм" функций из пространств Бесова $B_{p, q}^{s}\left(\mathbb{R}^{n}\right)$ и их однородных аналогов $\dot{B}_{p, q}^{s}\left(\mathbb{R}^{n}\right)$. Чтобы дать определение этих пространств введем в рассмотрение функцию $\varphi \in \mathscr{S}\left(\mathbb{R}^{n}\right)$ такую, что $\operatorname{supp} \varphi \subset\left\{\xi \in \mathbb{R}^{n}: 1 / 2 \leqslant|\xi| \leqslant 2\right\}, \varphi(\xi)>0$ при $1 / 2<$ $|\xi|<2$ и

$$
\sum_{k=-\infty}^{+\infty} \varphi\left(2^{-k} \xi\right)=1, \quad \xi \neq 0
$$

Введем также функции $\varphi_{k}$ и $\psi$ соотношениями

$$
\mathscr{F} \varphi_{k}(\xi)=\varphi\left(2^{-k} \xi\right), \quad \mathscr{F} \psi(\xi)=1-\sum_{k=1}^{\infty} \varphi\left(2^{-k} \xi\right) .
$$

Пусть $s \in \mathbb{R}, 0<p, q \leqslant \infty$. Будем говорить, что $f \in \mathscr{S}^{\prime}\left(\mathbb{R}^{n}\right)$ принадлежит (неоднородному) пространству Бесова $B_{p, q}^{s}$, если

$$
\|f\|_{B_{p, q}^{s}}=\|\psi * f\|_{p}+\left(\sum_{k=1}^{\infty} 2^{s q k}\left\|\varphi_{k} * f\right\|_{p}^{q}\right)^{\frac{1}{q}}<\infty
$$

с обычной модификацией при $q=\infty$, т. е.

$$
\|f\|_{B_{p, \infty}^{s}}=\|\psi * f\|_{p}+\sup _{k \geqslant 1} 2^{s k}\left\|\varphi_{k} * f\right\|_{p} .
$$

Для того чтобы дать определение однородных пространств Бесова, напомним, что

$$
\dot{\mathscr{S}}\left(\mathbb{R}^{n}\right)=\left\{\varphi \in \mathscr{S}\left(\mathbb{R}^{n}\right):\left(D^{\nu} \widehat{\varphi}\right)(0)=0 \forall \nu \in \mathbb{N}^{n} \cup\{0\}\right\},
$$

а $\dot{\mathscr{S}}^{\prime}\left(\mathbb{R}^{n}\right)$ - пространство всех непрерывных линейных функционалов на $\dot{\mathscr{S}}\left(\mathbb{R}^{n}\right)$.

Будем говорить, что $f \in \dot{\mathscr{S}}^{\prime}\left(\mathbb{R}^{n}\right)$ принадлежит однородному пространству Бесова $\dot{B}_{p, q}^{s}$, если

$$
\|f\|_{\dot{B}_{p, q}^{s}}=\left(\sum_{k=-\infty}^{+\infty} 2^{s q k}\left\|\varphi_{k} * f\right\|_{p}^{q}\right)^{\frac{1}{q}}<\infty
$$

(с обычной модификацией при $q=\infty$ ).

Мы будем рассматривать также пространства бесселевых (или риссовых) потенциалов $H_{p}^{s}\left(\mathbb{R}^{n}\right)$ (или $\left.\dot{H}_{p}^{s}\left(\mathbb{R}^{n}\right)\right)$. Напомним, что оператор $J^{s}=(I-\Delta)^{s / 2}$ $\left(\right.$ или $I^{s}=(-\Delta)^{s / 2}$ ) называют потенииалом Бесселя (или потенииалом Pисса). Здесь $I$ - единичный оператор, а $\Delta$ - оператор Лапласа.

Пусть $1<p<\infty$ и $s \in \mathbb{R}$. Будем говорить, что $f \in \mathscr{S}^{\prime}\left(\mathbb{R}^{n}\right)\left(\right.$ или $\left.f \in \dot{\mathscr{S}}^{\prime}\left(\mathbb{R}^{n}\right)\right)$ принадлежит пространству $H_{p}^{s}$ (или $\dot{H}_{p}^{s}$ ), если

$$
\left.\|f\|_{H_{p}^{s}}=\left\|J^{s} f\right\|_{p}<\infty \quad \text { (или }\|f\|_{\dot{H}_{p}^{s}}=\left\|I^{s} f\right\|_{p}<\infty\right) .
$$


Отметим, что данные пространства называют еще лебеговыми или лиувиллевыми пространствами.

Напомним хорошо известную связь между пространствами $H_{p}^{s}$ и обычными пространствами Соболева $W_{p}^{k}$ (см., например, [22, с. 116]). Пусть $1<p<\infty$ и $k \in \mathbb{N} \cup\{0\}$. Тогда

$$
H_{p}^{k}\left(\mathbb{R}^{n}\right)=W_{p}^{k}\left(\mathbb{R}^{n}\right)=\left\{f \in \mathscr{S}^{\prime}\left(\mathbb{R}^{n}\right):\|f\|_{W_{p}^{k}}^{*}<\infty\right\}
$$

где

$$
\|f\|_{W_{p}^{k}}^{*}=\|f\|_{p}+\sum_{j=1}^{n}\left\|\frac{\partial^{k} f}{\partial x_{j}^{k}}\right\|_{p}
$$

- эквивалентная норма в $H_{p}^{k}$ и $W_{p}^{k}$. Здесь и далее $W_{p}^{0}=L_{p}$.

Аналогичная связь имеет место между однородными пространствами $\dot{H}_{p}^{s}$ и $\dot{W}_{p}^{k}$ (см., например, [22, с. 343]).

Далее всюду через $C$ обозначены положительные константы, зависящие от указанных параметров, а через $A$ - некоторые конечные константы. Полагаем также $\frac{\infty}{\infty}=1$ и $\frac{0}{0}=0$.

\section{§ 3. Формулировка основных результатов}

Для формулировки основных результатов нам понадобится вспомогательная функция $\eta \in C^{\infty}\left(\mathbb{R}^{n}\right)$ такая, что $\operatorname{supp} \eta \subset\left\{\xi \in \mathbb{R}^{n}: 1 / 4 \leqslant|\xi| \leqslant 4\right\}, 0 \leqslant \eta(\xi) \leqslant 1$, для всех $\xi \in \mathbb{R}^{n}$ и $\eta(\xi)=1$ при $1 / 2 \leqslant|\xi| \leqslant 2$. Далее будем говорить, что функция $m$ принадлежит локально пространству $X$, если $m(\delta \cdot) \eta \in X$ для любого $\delta>0$.

Tеорема 1. Пусть $0<p<1,0<q, r \leqslant \infty, s>n(1 / p-1 / 2)$ и функция $m$ принадлежит локально пространству $L_{q} \cap B_{r, \infty}^{s}$. Предположим, что $q=r=2$ или

$$
\frac{1-\theta}{q}+\frac{\theta}{r}<\frac{1}{2}, \quad \theta=\frac{n}{s}\left(\frac{1}{p}-\frac{1}{2}\right) .
$$

Если, дополнительно,

$$
\sup _{\delta>0}\|m(\delta \cdot) \eta\|_{L_{q}}^{1-\theta}\|m(\delta \cdot) \eta\|_{B_{r, \infty}^{s}}^{\theta} \leqslant A
$$

mo $m \in \mathscr{M}\left(H_{p}\right) u\|m\|_{\mathscr{M}\left(H_{p}\right)} \leqslant C A$.

В пространствах $H_{1}\left(\mathbb{R}^{n}\right)$ и $L_{p}\left(\mathbb{R}^{n}\right), 1<p<2$, имеет место следующий более слабый аналог теоремы 1.

Теорема 2. Пусть $1 \leqslant p<2,0<q, r \leqslant \infty, s>\sigma>n(1 / p-1 / 2) u$ ограниченная функиия $m$ принадлежит локально пространству $B_{r, \infty}^{s}$. Предположим, что $1 / q=1 / r=1 / p-1 / 2$ или

$$
\frac{1-\theta}{q}+\frac{\theta}{r}<\frac{1}{p}-\frac{1}{2}, \quad \theta=\frac{\sigma}{s} .
$$


Если, дополнительно,

$$
\sup _{\delta>0}\|m(\delta \cdot) \eta\|_{L_{q}}^{1-\theta}\|m(\delta \cdot) \eta\|_{B_{r, \infty}^{s}}^{\theta} \leqslant A,
$$

mo $m \in \mathscr{M}\left(H_{p}\right) u\|m\|_{\mathscr{M}\left(H_{p}\right)} \leqslant C A$.

Используя теорему 1 , нетрудно проверить, что функция $m_{\alpha, \beta}$ (см. (21) далее) принадлежит пространству $\mathscr{M}\left(H_{p}\right), 0<p<1$, если $\beta \geqslant \alpha n(1 / p-1 / 2)$ (ср. с леммой 5 , приведенной ниже). С помощью теоремы 2 аналог данного результата для $1 \leqslant p<2$ можно получить только при $\beta>\alpha n(1 / p-1 / 2)$. Если $\beta=\alpha n(1 / p-1 / 2)$, то теоремы 2 , в отличие от теоремы 1 , уже не достаточно.

В следующих двух теоремах мы устраняем такой недостаток, однако для этого нам приходится брать $q=\infty$, если $s>n / 2$, и, дополнительно, $r=\infty$, если $s>n(1 / p-1 / 2)$.

Теорема 3. Пусть $1 \leqslant p<2,0<r \leqslant \infty, s>n / \min (r, 2)$ и функиия $m$ принадлежит локально пространству $L_{\infty} \cap B_{r, \infty}^{s}$. Eсли, дополнительно,

$$
\sup _{\delta>0}\|m(\delta \cdot) \eta\|_{L_{\infty}}^{1-\theta}\|m(\delta \cdot) \eta\|_{B_{r, \infty}^{s}}^{\theta} \leqslant A, \quad \theta=\frac{n}{s}\left(\frac{1}{p}-\frac{1}{2}\right),
$$

mo $m \in \mathscr{M}\left(H_{p}\right) u\|m\|_{\mathscr{M}\left(H_{p}\right)} \leqslant C A$.

Теорема 4. Пустъ $1<p<2, s>n(1 / p-1 / 2)$ и функиия $m$ принадлежит локально пространству $B_{\infty, \infty}^{s}$. Если, дополнительно,

$$
\sup _{\delta>0}\|m(\delta \cdot) \eta\|_{L_{\infty}}^{1-\theta}\|m(\delta \cdot) \eta\|_{B_{\infty}^{s}, \infty}^{\theta} \leqslant A, \quad \theta=\frac{n}{s}\left(\frac{1}{p}-\frac{1}{2}\right),
$$

mo $m \in \mathscr{M}\left(L_{p}\right) u\|m\|_{\mathscr{M}\left(L_{p}\right)} \leqslant C A$.

В следующих двух предложениях показана точность теорем 1-4.

ПрЕДЛОЖЕНИЕ 1. Пусть $0<p<2,1 \leqslant q, r \leqslant \infty u s>n(1 / p-1 / 2)$. Если $\theta^{*}<n(1 / p-1 / 2) / s$, то найдется функиия $m \in L_{\infty}\left(\mathbb{R}^{n}\right) \cap C^{\infty}\left(\mathbb{R}^{n}\right)$ такая, что

$$
\sup _{\delta>0}\|m(\delta \cdot) \eta\|_{L_{q}}^{1-\theta^{*}}\|m(\delta \cdot) \eta\|_{B_{r, \infty}^{s}}^{\theta^{*}}<\infty
$$

но $m \notin \mathscr{M}\left(H_{p}\right)$.

ПреДЛОЖЕНИЕ 2. Пусть $0<p<2,1 \leqslant q, r \leqslant \infty, s>n(1 / p-1 / 2) u$ $\theta=n(1 / p-1 / 2) / s$. Если

$$
\begin{gathered}
\frac{1-\theta}{q}+\frac{\theta}{r}>\frac{1}{2} \quad \text { nрu } \quad 0<p<1, \\
\frac{1-\theta}{q}+\frac{\theta}{r} \geqslant \frac{1}{p}-\frac{1}{2} \quad \text { npu } \quad 1 \leqslant p<2,
\end{gathered}
$$

то найдется функиця $m \in L_{\infty}\left(\mathbb{R}^{n}\right) \cap C^{\infty}\left(\mathbb{R}^{n}\right)$ такая, что

$$
\sup _{\delta>0}\|m(\delta \cdot) \eta\|_{L_{q}}^{1-\theta}\|m(\delta \cdot) \eta\|_{B_{r, \infty}^{s}}^{\theta}<\infty
$$

но $m \notin \mathscr{M}\left(H_{p}\right)$. 
Перейдем к рассмотрению достаточных условий для мультипликаторов Фурье в пространствах $L_{1}\left(\mathbb{R}^{n}\right)$ (или $\left.L_{\infty}\right)$. Прежде чем сформулировать следующий результат, рассмотрим класс функций, представимых в виде абсолютно сходящегося интеграла Фурье:

$$
W_{0}\left(\mathbb{R}^{n}\right)=\left\{f: f(\xi)=\int_{\mathbb{R}^{n}} e^{-i(x, \xi)} g(x) d x,\|f\|_{W_{0}}=\|g\|_{1}<\infty\right\},
$$

который называют алгеброй Винера или колъцом Винера. Хорошо известно (см., например, [18, с. 113]), что принадлежность функции $f$ пространству $W_{0}\left(\mathbb{R}^{n}\right)$ делает ее мультипликатором Фурье в пространстве $L_{1}\left(\mathbb{R}^{n}\right)$ и, следовательно, мультипликатором Фурье в $L_{p}\left(\mathbb{R}^{n}\right)$ при $1<p<\infty$. Таким образом, следующую теорему можно рассматривать как достаточное условие для мультипликаторов Фурье в $L_{p}\left(\mathbb{R}^{n}\right)$ при всех $1 \leqslant p \leqslant \infty$.

Tеорема 5. Пусть $0<q, r \leqslant \infty, \sigma<n / q, s>n / r, s>\sigma u f \in C_{0}\left(\mathbb{R}^{n}\right)$. Предположим, что $q=r=2$ или

$$
\frac{1-\theta}{q}+\frac{\theta}{r}>\frac{1}{2}, \quad \theta=\frac{n / 2-\sigma}{s-\sigma} .
$$

Если, дополнительно, $f \in \dot{B}_{q, \infty}^{\sigma} \cap \dot{B}_{r, \infty}^{s}$, mo $f \in W_{0}\left(\mathbb{R}^{n}\right) u$

$$
\|f\|_{W_{0}} \leqslant C\|f\|_{\dot{B}_{q, \infty}^{\sigma}}^{1-\delta}\|f\|_{\dot{B}_{r, \infty}^{s}}^{\delta}, \quad \delta=\frac{n / q-\sigma}{s-\sigma+n(1 / q-1 / r)},
$$

әде $C$ - константа, не зависящая от $f$.

Как и предыдущие теоремы, данный результат является точным.

ПреДЛОЖеНИЕ 3. Пусть $1 \leqslant q, r \leqslant \infty, \sigma, s \in \mathbb{N} \cup\{0\}, \sigma<n / q, s>n / r u$ $s>\sigma$. Eслu

$$
\frac{1-\theta}{q}+\frac{\theta}{r}<\frac{1}{2}, \quad \theta=\frac{n / 2-\sigma}{s-\sigma},
$$

то найдется функиия $f \in C_{0} \cap \dot{B}_{q, \infty}^{\sigma} \cap \dot{B}_{r, \infty}^{s}$, но $f \notin W_{0}\left(\mathbb{R}^{n}\right)$.

\section{§ 4. Некоторые следствия и замечания}

Из теорем 1-4, учитывая непрерывное вложение $H_{r}^{s} \subset B_{r, \infty}^{s}$ (см., например, [22, с. 59, 60]), получаем

СлЕДСТвиЕ 1. Пусть $0<p<2,1<r<\infty, s>\frac{2 n}{\min (r, 2)}\left(\frac{1}{\min (p, 1)}-\frac{1}{2}\right) u$ функиия $m$ принадлежит локально пространству $L_{\infty} \cap H_{r}^{s}$. Если

$$
\sup _{\delta>0}\|m(\delta \cdot) \eta\|_{L_{\infty}}^{1-\theta}\|m(\delta \cdot) \eta\|_{H_{r}^{s}}^{\theta} \leqslant A
$$

mo $m \in \mathscr{M}\left(H_{p}\right) u\|m\|_{\mathscr{M}\left(H_{p}\right)} \leqslant C A$.

Легко видеть, что следствие 1 является усилением дробного варианта теоремы Хёрмандера (см. [6] и [16]), а также усилением достаточных условий для мультипликаторов из [7]. 
Для формулировки следующего утверждения напомним определение пространства Зигмунда $\mathscr{C}^{s}\left(\mathbb{R}^{n}\right)$. Пусть $s=k+\sigma$, где $k \in \mathbb{N} \cup\{0\}$ и $0<\sigma \leqslant 1$. Положим

$$
\begin{aligned}
\mathscr{C}^{s}\left(\mathbb{R}^{n}\right)=\left\{f \in C^{k}\left(\mathbb{R}^{n}\right):\|f\|_{\mathscr{C}^{s}\left(\mathbb{R}^{n}\right)}=\right. & \sum_{|\nu|_{1} \leqslant k}\left\|D^{\nu} f\right\|_{\infty} \\
& \left.+\sum_{|\nu|_{1}=k} \sup _{h \in \mathbb{R}^{n}}|h|^{-\sigma}\left\|\Delta_{h}^{2} D^{\nu} f\right\|_{\infty}<\infty\right\},
\end{aligned}
$$

где $\Delta_{h}^{2} f$ - вторая разность функции $f$ с шагом $h$.

Из теорем 1-4, учитывая, что $\mathscr{C}^{s}\left(\mathbb{R}^{n}\right)=B_{\infty, \infty}^{s}\left(\mathbb{R}^{n}\right), s>0$ (см., например, [22, c. 118]), получаем следующее усиление теоремы G из работы [8].

СлеДСтвиЕ 2. Пусть $0<p<2, s>n(1 / p-1 / 2)$ и функиия $m$ принадлежит локально пространству $\mathscr{C}^{s}\left(\mathbb{R}^{n}\right)$. Если, дополнительно,

$$
\sup _{\delta>0}\|m(\delta \cdot) \eta\|_{L_{\infty}}^{1-\theta}\|m(\delta \cdot) \eta\|_{\mathscr{C} s}^{\theta} \leqslant A, \quad \theta=\frac{n}{s}\left(\frac{1}{p}-\frac{1}{2}\right),
$$

mo $m \in \mathscr{M}\left(L_{p}\right) u\|m\|_{\mathscr{M}\left(L_{p}\right)} \leqslant C A$.

Для классов $W_{0}\left(\mathbb{R}^{n}\right)$ из теоремы 5, используя непрерывное вложение $\dot{H}_{r}^{s} \subset$ $\dot{B}_{r, \infty}^{s}$ (см., например, [19, с. 188]), получаем

СлеДСтвие 3. Пусть $0<q \leqslant \infty, 1<r<\infty, s>n / r u f \in C_{0}\left(\mathbb{R}^{n}\right)$. Предположим, что $q=r=2$ или

$$
\left(1-\frac{n}{2 s}\right) \frac{1}{q}+\left(\frac{n}{2 s}\right) \frac{1}{r}>\frac{1}{2} .
$$

Если, дополнительно, $f \in L_{q} \cap \dot{H}_{r}^{s}$, mо $f \in W_{0}\left(\mathbb{R}^{n}\right)$ u

$$
\|f\|_{W_{0}} \leqslant C\|f\|_{L_{q}}^{1-\delta}\|f\|_{\dot{H}_{r}^{s}}^{\delta}, \quad \delta=\frac{n / q}{s+n(1 / q-1 / r)},
$$

где $C$ - константа, не зависящая от $f$.

Легко видеть, что при $s=n=1$ мы получаем основной результат работы [16], а при $q=r=2$ - известную теорему типа Бёрлинга (см. [20] и [21]).

Следствия 1-3 являются точными. Доказательство этого факта аналогично доказательству предложений 1-3.

Из предложения 2 следует, что утверждение теоремы 3 с условием (9) вместо (12) и $q=s=2$ уже не выполняется. Однако если $p=1$, то при некотором видоизменении условия $(12) L_{\infty}$-норму функции $m(\delta \cdot) \eta$ можно заменить $L_{2}$-нормой. Так, применяя теорему 2.2 из [12], в которой показано, что $m \in \mathscr{M}\left(H_{1}\right)$, если $\sum_{j=-\infty}^{+\infty}\left\|\mathscr{F}\left(m\left(2^{j} \cdot\right) \eta\right)\right\|_{1}^{2}<\infty$, а также используя теорему 5 , получаем следующее достаточное условие для мультипликаторов в $H_{1}\left(\mathbb{R}^{n}\right)$. 
Tеорема 6. Пусть $s>n / 2$. Если

$$
\sum_{j=-\infty}^{+\infty}\left\|m\left(2^{j} \cdot\right) \eta\right\|_{L_{2}}^{1-\frac{n}{2 s}}\left\|m\left(2^{j} \cdot\right) \eta\right\|_{\dot{B}_{2, \infty}^{s}}^{\frac{n}{2 s}}<\infty
$$

mo $m \in \mathscr{M}\left(H_{1}\right)$.

Отметим, что теорема 6 , с одной стороны, является усилением теорем 3,4 , поскольку $\left\|m_{2^{j}}\right\|_{q} \leqslant C\left\|m_{2^{j}}\right\|_{\infty}$, с другой стороны, как и теорема 2 , уже не годится для полного исследования функций вида (3).

\section{§ 5. Вспомогательные утверждения}

Приведем сначала некоторые хорошо известные факты о пространствах Бесова. Мы будем ссылаться, в основном, на монографии [22] и [23]. Необходимую информацию о пространствах Бесова можно найти также в [19], [24] и $[25]$.

Прежде всего дадим одно эквивалентное определение однородных пространств Бесова (см., например, в [22, с. 144]). Далее если $f$ - произвольная функция на $\mathbb{R}^{n}$ и $h \in \mathbb{R}^{n}$, то

$$
\Delta_{h}^{1} f(x)=f(x+h)-f(x), \quad \Delta_{h}^{l} f(x)=\Delta_{h}^{l}\left(\Delta_{h}^{l-1} f(x)\right) .
$$

Лемма 1. Пусть $0<p, q \leqslant \infty u s>n(1 / \min (p, 1)-1)$. Если $l \in \mathbb{N} u l>s$, то величина

$$
\|f\|_{B_{p, q}^{s}}^{*}=\|f\|_{p}+\left(\int_{\mathbb{R}^{n}}|h|^{-s q}\left\|\Delta_{h}^{l} f\right\|_{p}^{q} \frac{d h}{|h|^{n}}\right)^{\frac{1}{q}}
$$

является эквивалентной квазинормой в $B_{p, q}^{s}$ (с обычной модификацией при $q=\infty$, m.e. $\left.\|f\|_{B_{p, \infty}^{s}}^{*}=\|f\|_{p}+\sup _{h \in \mathbb{R}^{n}}|h|^{-s q}\left\|\Delta_{h}^{l} f\right\|_{p}\right)$.

Мы будем часто использовать следующие вложения для пространств Бесова.

Лемма 2. (i) Пусть $0<p \leqslant \infty, 0<q_{0} \leqslant q_{1} \leqslant \infty u s \in \mathbb{R}$. Тогда

$$
B_{p, q_{0}}^{s} \subset B_{p, q_{1}}^{s} \quad\left(\dot{B}_{p, q_{0}}^{s} \subset \dot{B}_{p, q_{1}}^{s}\right) .
$$

(ii) Пусть $0<p_{0} \leqslant p_{1} \leqslant \infty, 0<q \leqslant \infty, s_{0}, s_{1} \in \mathbb{R}, s_{1} \leqslant s_{0} u s_{0}-n / p_{0}=$ $s_{1}-n / p_{1}$. Тогда

$$
B_{p_{0}, q}^{s_{0}} \subset B_{p_{1}, q}^{s_{1}} \quad\left(\dot{B}_{p_{0}, q}^{s_{0}} \subset \dot{B}_{p_{1}, q}^{s_{1}}\right) .
$$

(iii) Пусть $0<p, q_{0}, q_{1} \leqslant \infty, s \in \mathbb{R} u \varepsilon>0$. Тогда

$$
B_{p, q_{0}}^{s+\varepsilon} \subset B_{p, q_{1}}^{s}
$$

Здесь и далее через $\subset$ обозначено непрерывное вложение.

Напомним также хорошо известные вложения для пространств $B_{p, q}^{k}, W_{p}^{k}, C^{k}$ и для их однородных аналогов. 
Лемма 3. Пусть $k \in \mathbb{N} \cup\{0\} u 1 \leqslant p<\infty$. Тогда

$$
\begin{array}{cl}
B_{p, 1}^{k} \subset W_{p}^{k} \subset B_{p, \infty}^{k} & \left(\dot{B}_{p, 1}^{k} \subset \dot{W}_{p}^{k} \subset \dot{B}_{p, \infty}^{k}\right), \\
B_{\infty, 1}^{k} \subset C^{k} \subset B_{\infty, \infty}^{k} & \left(\dot{B}_{\infty, 1}^{k} \subset \dot{C}^{k} \subset \dot{B}_{\infty, \infty}^{k}\right) .
\end{array}
$$

ДокАЗАТЕЛЬство лемм 2, 3 см., например, в [22, гл. 2, гл. 5] и [23, гл. 3, гл. 11].

Приведем свойство дилатации для пространств $\dot{B}_{p, q}^{s}$ (см., например, $[22$, c. 339]).

Лемма 4. Пустъ $0<p, q \leqslant \infty, \lambda>0 u f \in \dot{B}_{p, q}^{s}\left(\mathbb{R}^{n}\right)$. Тогда

$$
\|f(\lambda \cdot)\|_{\dot{B}_{p, q}^{s}} \leqslant C \lambda^{s-\frac{n}{p}}\|f\|_{\dot{B}_{p, q}^{s}}
$$

где $C$ - некоторая положительная константа, не зависящая от $f u \lambda$.

Для доказательства точности условий теорем 1-5 нам понадобится известная функция-мультипликатор

$$
m_{\alpha, \beta}(\xi)=\rho(\xi) \frac{e^{i|\xi|^{\alpha}}}{|\xi|^{\beta}}, \quad \alpha, \beta>0,
$$

где $\rho \in C^{\infty}\left(\mathbb{R}^{n}\right), \rho(\xi)=0$ при $|\xi|<1$ и $\rho(\xi)=1$ при $|\xi| \geqslant 2$. Отметим, что функция $m_{\alpha, \beta}$ была предметом специального изучения в работах [26], [5], [7] и [8] (см. также ссылки в [18, гл. IV, п. 7.4]). Доказательство утверждений следующей леммы см., например, в [8, предложение 5.1].

Лемма 5. Пусть $\alpha, \beta>0$ и $\alpha \neq 1$. Справедливы следующие утверждения:

(i) $m_{\alpha, \beta} \in \mathscr{M}\left(H_{p}\right)$ тогда и только тогда, когда $\beta \geqslant n(1 / p-1 / 2) \alpha$;

(ii) $m_{\alpha, \beta} \in W_{0}\left(\mathbb{R}^{n}\right)$ тогда и только тогда, когда $\beta>n \alpha / 2$.

Заметим, что достаточную часть утверждений леммы 5 можно легко получить из следствий 1,3 .

Лемма 6. Пусть $0<\alpha<2 u 2<\beta \leqslant \infty$. Тогда

$$
\|f\|_{2} \leqslant\|f\|_{\alpha}^{\frac{\alpha}{2}\left(\frac{\beta-2}{\beta-\alpha}\right)}\|f\|_{\beta}^{\frac{\beta}{2}\left(\frac{2-\alpha}{\beta-\alpha}\right)} .
$$

Данная лемма является простым следствием из неравенства Гёльдера.

\section{§ 6. Доказательство основных результатов}

Всюду далее для удобства положим $m_{\delta}(\xi)=m(\delta \xi) \eta(\xi)$.

ДоКАЗАТЕЛЬСТво ТЕоремы 1 основано на следующем достаточном условии для мультипликаторов Фурье в пространстве $H_{p}\left(\mathbb{R}^{n}\right)$ при $0<p<1$ (см. [9, c. 20]).

Лемма 7. Пусть $0<p<1 u \sigma=n(1 / p-1 / 2)$. Если

$$
\sup _{j \in \mathbb{Z}}\left\|m_{2^{j}}\right\|_{B_{2, p}^{\sigma}} \leqslant A,
$$

mo $m \in \mathscr{M}\left(H_{p}\right) u\|m\|_{\mathscr{M}\left(H_{p}\right)} \leqslant C A$. 
Покажем сначала, что $\left\|m_{2^{j}}\right\|_{2} \leqslant C A$. Действительно, выберем $q^{*}<q$ так, чтобы

$$
\frac{1-\theta}{q^{*}}+\frac{\theta}{r}=\frac{1}{2} \text {. }
$$

Тогда из неравенства Гёльдера и леммы 1, учитывая, что $\operatorname{supp} m_{2^{j}} \subset\{\xi \in$ $\left.\mathbb{R}^{n}:|\xi| \leqslant 4\right\}$ и $s>n / r$, получаем

$$
\left\|m_{2^{j}}\right\|_{2} \leqslant\left\|m_{2^{j}}\right\|_{q^{*}}^{1-\theta}\left\|m_{2^{j}}\right\|_{r}^{\theta} \leqslant C\left\|m_{2^{j}}\right\|_{q}^{1-\theta}\left\|m_{2^{j}}\right\|_{B_{r, \infty}^{s}}^{\theta} \leqslant C A .
$$

Таким образом, согласно лемме 7 для доказательства теоремы достаточно проверить, что

$$
I_{j}=\int_{\mathbb{R}^{n}}|h|^{-\sigma p}\left\|\Delta_{h}^{l} m_{2^{j}}\right\|_{2}^{p} \frac{d h}{|h|^{n}} \leqslant C A^{p}, \quad j \in \mathbb{Z},
$$

где $l>\sigma$. Для этого запишем $I_{j}$ в виде

$$
I_{j}=\int_{|h| \leqslant \gamma_{j}}+\int_{|h|>\gamma_{j}}=I_{j}^{(1)}+I_{j}^{(2)},
$$

где $\gamma_{j}=\left(\left\|m_{2^{j}}\right\|_{q}\left\|m_{2^{j}}\right\|_{B_{r, \infty}^{s}}^{-1}\right)^{1 / s}$, и покажем, что

$$
\sup _{j \in \mathbb{Z}} I_{j}^{(i)} \leqslant C A^{p}, \quad i=1,2 .
$$

Прежде всего заметим, что при $0<q \leqslant \infty$ и $2 \leqslant r \leqslant \infty$

$$
\gamma_{j} \leqslant C
$$

где $C$ - константа, зависящая только от $q, r, s$ и $n$. Действительно, если $q<r$, то, применяя неравенство Гёльдера, а затем вложения $B_{r, \infty}^{s} \subset B_{r, 1}^{0} \subset L_{r}$ (см. лемму 2, (iii) и лемму 3), имеем

$$
\left\|m_{2^{j}}\right\|_{q} \leqslant C\left\|m_{2^{j}}\right\|_{r} \leqslant C\left\|m_{2^{j}}\right\|_{B_{r, \infty}^{s}} .
$$

Отсюда сразу следует неравенство (24). Если $q \geqslant r$, то, учитывая, что $s_{1}=$ $s-n(1 / r-1 / q)>0$, и применяя лемму 1 и лемму 2, (ii), имеем

$$
\left\|m_{2^{j}}\right\|_{q} \leqslant C\left\|m_{2^{j}}\right\|_{B_{q, \infty}^{s_{1}}} \leqslant C\left\|m_{2^{j}}\right\|_{B_{r, \infty}^{s}} .
$$

Отсюда также получаем (24).

Перейдем к доказательству неравенств (23).

1) Рассмотрим сначала случай $2 \leqslant q, r \leqslant \infty$. Используя неравенство Гёльдера, а также лемму 1, находим

$$
\begin{aligned}
I_{j}^{(1)} & \leqslant C \int_{|h| \leqslant \gamma_{j}}|h|^{-\sigma p}\left\|\Delta_{h}^{l} m_{2^{j}}\right\|_{r}^{p} \frac{d h}{|h|^{n}} \\
& \leqslant C \int_{|h| \leqslant \gamma_{j}}|h|^{(s-\sigma) p}\left(\sup _{t \in \mathbb{R}^{n}} \frac{\left\|\Delta_{t}^{l} m_{2^{j}}\right\|_{r}}{|t|^{s}}\right)^{p} \frac{d h}{|h|^{n}} \leqslant C \gamma_{j}^{(s-\sigma) p}\left\|m_{2^{j}}\right\|_{B_{r, \infty}^{s}}^{p}=C A^{p} .
\end{aligned}
$$


Чтобы оценить интеграл $I_{j}^{(2)}$, достаточно воспользоваться неравенством Гёльдера и следующим простым соотношением:

$$
\left\|\Delta_{h}^{l} m_{2^{j}}\right\|_{q} \leqslant C\left\|m_{2^{j}}\right\|_{q}, \quad 0<q \leqslant \infty
$$

где $C$ - константа, зависящая только от $l$ и $q$ :

$$
I_{j}^{(2)} \leqslant C\left\|m_{2^{j}}\right\|_{2}^{p} \int_{|h| \geqslant \gamma_{j}} \frac{d h}{|h|^{\sigma p+n}} \leqslant C\left\|m_{2^{j}}\right\|_{q}^{p} \gamma_{j}^{-\sigma p}=C A^{p} .
$$

Объединяя (25) и (27), получаем (23) для данных $q$ и $r$.

2) Теперь рассмотрим случай $0<q<2<r \leqslant \infty$. Интеграл $I_{j}^{(1)}$ оценивается так же, как и в (25). Рассмотрим $I_{j}^{(2)}$. Положим $\alpha=q, \beta=r$ в лемме 6 и обозначим $\lambda=\frac{r}{2}\left(\frac{2-q}{r-q}\right)$. Учитывая, что неравенство $\lambda<\theta$ эквивалентно неравенству (8), а также используя неравенство (26) и лемму 1, находим

$$
\begin{aligned}
I_{j}^{(2)} & \leqslant \int_{|h| \geqslant \gamma_{j}}|h|^{-\sigma p}\left\|\Delta_{h}^{l} m_{2^{j}}\right\|_{q}^{(1-\lambda) p}\left\|\Delta_{h}^{l} m_{2^{j}}\right\|_{r}^{\lambda p} \frac{d h}{|h|^{n}} \\
& \leqslant C\left\|m_{2^{j}}\right\|_{q}^{(1-\lambda) p} \int_{|h| \geqslant \gamma_{j}}|h|^{(s \lambda-\sigma) p}\left(\sup _{t \in \mathbb{R}^{n}} \frac{\left\|\Delta_{t}^{l} m_{2^{j}}\right\|_{r}}{|t|^{s}}\right)^{\lambda p} \frac{d h}{|h|^{n}} \\
& \leqslant C \gamma_{j}^{(s \lambda-\sigma) p}\left\|m_{2^{j}}\right\|_{q}^{(1-\lambda) p}\left\|m_{2^{j}}\right\|_{B_{r, \infty}^{s p}}^{\lambda p}=C A^{p} .
\end{aligned}
$$

Таким образом, объединяя (28) и (25), мы также получаем (23).

3) Случай $2<q \leqslant \infty, 0<r<2$ доказывается аналогично. Теорема доказана.

ДокАЗАТЕЛЬСтво теОРемы 2. Из [6, теоремы 4.6, 4.7] следует, что если $1 \leqslant p<2, \sigma>n(1 / p-1 / 2)$ и $1 / t=1 / p-1 / 2$, а функция $m \in L_{\infty}\left(\mathbb{R}^{n}\right)$ удовлетворяет неравенству

$$
\sup _{\delta>0}\left\|m_{\delta}\right\|_{H_{t}^{\sigma}} \leqslant A
$$

то $m \in \mathscr{M}\left(H_{p}\right)$ и $\|m\|_{\mathscr{M}\left(H_{p}\right)} \leqslant C A$.

Используя вложение $B_{t, 1}^{\sigma} \subset H_{t}^{\sigma}$ (см., например, $[22$, с. 59,60$\left.]\right)$, мы получаем, что $m \in \mathscr{M}\left(H_{p}\right)$, если вместо (29) выполнено неравенство

$$
\sup _{\delta>0}\left\|m_{\delta}\right\|_{B_{t, 1}^{\sigma}} \leqslant A
$$

Таким образом, повторяя приведенное выше доказательство теоремы 1 , нетрудно убедиться в справедливости теоремы.

ДоКАЗАТЕЛЬСТВо тЕОРЕмЫ 3 основано на методе комплексной интерполяции (см., например, [6, с. 151, 152] или [22, с. 86-90]).

Выберем $0<p_{0}<1$ так, чтобы $s>n\left(1 / p_{0}-1 / 2\right)$, и определим число $\gamma \in(0,1)$ из равенства

$$
\frac{1}{p}=\frac{1-\gamma}{p_{0}}+\frac{\gamma}{2}
$$


Положим

$$
m(\xi, z)=\sum_{j \in \mathbb{Z}}\left\|m_{2^{j}}\right\|_{\infty}^{\frac{\gamma-z}{1-\gamma}} m(\xi) \eta\left(2^{-j} \xi\right), \quad 0 \leqslant \operatorname{Re} z \leqslant 1
$$

Проверим, что

$$
\begin{gathered}
\|m(\cdot, 1+i y)\|_{\mathscr{M}\left(L_{2}\right)} \leqslant C, \\
\|m(\cdot, i y)\|_{\mathscr{M}\left(H_{p_{0}}\right)} \leqslant C A^{\frac{1}{1-\gamma}} .
\end{gathered}
$$

Неравенство (30) вытекает из следующей простой оценки:

$$
|m(\xi, 1+i y)| \leqslant \sum_{j \in \mathbb{Z}} \chi_{\left\{2^{j-2} \leqslant|\xi| \leqslant 2^{j+2}\right\}}(\xi) \leqslant C
$$

(где $\chi_{E}$ - характеристическая функция множества $\left.E\right)$, и того, что $L_{\infty}=\mathscr{M}\left(L_{2}\right)$.

Докажем неравенство (31). Для этого мы воспользуемся теоремой 1. Заметим, что при $p_{0}$, достаточно близком к 1 , условие $(8)$ в теореме 1 будет выполнено.

Запишем функцию $m(\xi, i y)$ в следующем виде:

$$
m(\xi, i y)=\sum_{l=-3}^{3} m^{(l)}(\xi, i y)
$$

где

$$
m^{(l)}(\xi, i y)=\sum_{k \equiv l(\bmod 7)}\left\|m_{2^{k}}\right\|_{\infty}^{\frac{\gamma-i y}{1-\gamma}} m(\xi) \eta\left(2^{-k} \xi\right),
$$

и проверим, что

$$
\left\|m^{(l)}(\cdot, i y)\right\|_{\mathscr{M}\left(H_{p_{0}}\right)} \leqslant C A^{\frac{1}{1-\gamma}}, \quad l=-3, \ldots, 3 .
$$

Тогда, используя элементарные свойства мультипликаторов Фурье, мы, очевидно, будем иметь (31).

Заметим, что при фиксированных $l \in[-3,3]$ и $j \in \mathbb{Z}$ имеет место равенство

$$
m_{2^{j}}^{(l)}(\xi, i y)=\left\|m_{2^{k}}\right\|_{\infty}^{\frac{\gamma-i y}{1-\gamma}} m\left(2^{k-l} \xi\right) \eta\left(2^{-l} \xi\right) \eta(\xi), \quad k=j+l .
$$

Следовательно,

$$
\begin{aligned}
S_{j}^{(l)} & :=\left\|m_{2^{j}}^{(l)}(\cdot, i y)\right\|_{\infty}^{1-\theta_{0}}\left\|m_{2^{j}}^{(l)}(\cdot, i y)\right\|_{B_{r, \infty}^{s}}^{\theta_{0}} \\
& \leqslant C\left\|m_{2^{k}}\right\|_{\infty}^{\frac{\gamma}{1-\gamma}+1-\theta_{0}}\left\|m_{2^{k}} \eta\left(2^{l} \cdot\right)\right\|_{B_{r, \infty}^{s}}^{\theta_{0}} \leqslant C\left\|m_{2^{k}}\right\|_{\infty}^{\frac{\gamma}{1-\gamma}+1-\theta_{0}}\left\|m_{2^{k}}\right\|_{B_{r, \infty}^{s}}^{\theta_{0}},
\end{aligned}
$$

где $\theta_{0}=n\left(1 / p_{0}-1 / 2\right) / s$ и $k=j+l$. В последних двух неравенствах в (33) мы воспользовались леммой 1 и достаточным условием для поточечных мультипликаторов в пространствах Бесова (см., например, [22, с. 204]).

Продолжая оценивать (33), используя при этом соотношение (12) и учитывая, что $\theta_{0}-\frac{\gamma}{1-\gamma} \theta=\theta$, находим

$$
S_{j}^{(l)} \leqslant C A^{\frac{\gamma}{1-\gamma}}\left\|m_{2^{j+l}}\right\|_{\infty}^{1-\theta_{0}+\frac{\gamma}{1-\gamma} \theta_{0}}\left\|m_{2^{j+l}}\right\|_{B_{r, \infty}^{s}}^{\theta_{0}-\frac{\gamma}{1-\gamma} \theta} \leqslant C A^{\frac{1}{1-\gamma}} .
$$


Из последнего неравенства и теоремы 1 мы сразу получаем (32) и, следовательно, (31).

Таким образом, используя [6, теорема 3.4] и неравенства (30) и (31), получаем, что $m=m(\cdot, \gamma) \in \mathscr{M}\left(H_{p}\right)$ и $\|m\|_{\mathscr{M}\left(H_{p}\right)} \leqslant C A$. Теорема доказана.

ДоКАЗАТЕЛЬСТВо ТЕОРЕмЫ 4. Снова воспользуемся методом комплексной интерполяции.

Возьмем $1<p_{0}<2$ и $s_{0}>n / 2$ так, чтобы $s>s_{0}(1-\gamma)$, где число $\gamma \in(0,1)$ определяется из равенства

Введем функцию

$$
\frac{1}{p}=\frac{1-\gamma}{p_{0}}+\frac{\gamma}{2}
$$

$$
m(\xi, z)=\sum_{j \in \mathbb{Z}}\left\|m_{2^{j}}\right\|_{\infty}^{\frac{\gamma-z}{1-\gamma}} \psi\left(2^{-j} \xi\right)\left(J_{\lambda_{j}}^{(z-\gamma) s_{0}} m_{2^{j}}\right)\left(2^{-j} \xi\right), \quad 0 \leqslant \operatorname{Re} z \leqslant 1,
$$

где:

1) $\psi \in C^{\infty}\left(\mathbb{R}^{n}\right)$, supp $\psi \subset\left\{\xi \in \mathbb{R}^{n}: 1 / 8<|\xi|<8\right\}$ и $\psi(\xi)=1$, если $\xi \in \operatorname{supp} \eta$;

2) $J_{\lambda}^{z} f(\xi)=\left(J^{z} \tau_{\lambda} f\right)(\xi / \lambda)$, где $\tau_{\lambda} f=f(\lambda \cdot)$;

3) $\lambda_{j}=\left(\left\|m_{2^{j}}\right\|_{\infty}\left\|m_{2^{j}}\right\|_{\dot{B}_{\infty, \infty}^{s}}^{-1}\right)^{1 / s}$.

В силу интерполяционной теоремы 4.1 из [27, с. 231] для доказательства теоремы достаточно проверить, что

$$
\begin{gathered}
\|m(\cdot, 1+i y)\|_{\mathscr{M}\left(L_{2}\right)} \leqslant C(1+|y|)^{n+1}, \\
\|m(\cdot, i y)\|_{\mathscr{M}\left(L_{p_{0}}\right)} \leqslant C(1+|y|)^{n+1} A^{\frac{1}{1-\gamma}} .
\end{gathered}
$$

Докажем сначала (34). Для этого воспользуемся тем фактом, что функция

$$
g_{y}(\xi)=(1+|\xi|)^{-\varepsilon+i y}
$$

при любых $\varepsilon>0$ и $y \in \mathbb{R}$ является мультипликатором Фурье в пространстве $L_{\infty}\left(\mathbb{R}^{n}\right)$ и

$$
\left\|g_{y}\right\|_{\mathscr{M}\left(L_{\infty}\right)} \leqslant C(1+|y|)^{n+1},
$$

где $C$ - константа, не зависящая от $y$ (см., например, [6, лемма 4.2]).

Имеем

$$
\begin{aligned}
|m(\xi, 1+i y)| & \leqslant \sum_{k \in \mathbb{Z}} \psi\left(2^{-k} \xi\right) \sup _{j \in \mathbb{Z}}\left\|m_{2^{j}}\right\|_{\infty}^{\frac{\gamma-z}{1-\gamma}}\left\|J_{\lambda_{j}}^{(1-\gamma) s_{0}+i y s_{0}} m_{2^{j}}\right\|_{\infty} \\
& \leqslant C(1+|y|)^{n+1} \sup _{j \in \mathbb{Z}}\left\|m_{2^{j}}\right\|_{\infty}^{-1}\left\|J_{\lambda_{j}}^{(1-\gamma) s_{0}+\varepsilon} m_{2^{j}}\right\|_{\infty},
\end{aligned}
$$

где $\varepsilon-$ положительное число такое, что $s=(1-\gamma) s_{0}+2 \varepsilon$.

Далее, используя лемму 3 и лемму 2, (iii), свойство лифтинга для оператора $J^{s}$ (см., например, [22, с. 76$\left.]\right)$ и лемму 4, последовательно находим

$$
\begin{aligned}
\left\|J_{\lambda_{j}}^{(1-\gamma) s_{0}+\varepsilon} m_{2^{j}}\right\|_{\infty} & =\left\|J^{(1-\gamma) s_{0}+\varepsilon} \tau_{\lambda_{j}} m_{2^{j}}\right\|_{\infty} \\
& \leqslant C\left\|J^{(1-\gamma) s_{0}+\varepsilon} \tau_{\lambda_{j}} m_{2^{j}}\right\|_{B_{\infty, 1}^{0}} \leqslant C\left\|J^{(1-\gamma) s_{0}+\varepsilon} \tau_{\lambda_{j}} m_{2^{j}}\right\|_{B_{\infty, \infty}^{\varepsilon}} \\
& \leqslant C\left\|\tau_{\lambda_{j}} m_{2^{j}}\right\|_{B_{\infty, \infty}^{s}} \leqslant C\left\{\left\|\tau_{\lambda_{j}} m_{2^{j}}\right\|_{\infty}+\left\|\tau_{\lambda_{j}} m_{2^{j}}\right\|_{\dot{B}_{\infty, \infty}^{s}}\right\} \\
& \leqslant C\left\{\left\|m_{2^{j}}\right\|_{\infty}+\lambda_{j}^{s}\left\|m_{2^{j}}\right\|_{\dot{B}_{\infty, \infty}^{s}}\right\}=C\left\|m_{2^{j}}\right\|_{\infty}
\end{aligned}
$$


Таким образом, объединяя неравенства (37) и (38) и учитывая при этом, что $L_{\infty}=\mathscr{M}\left(L_{2}\right)$, мы сразу получаем $(34)$.

Теперь докажем неравенство (35). По аналогии с доказательством теоремы 3 запишем функцию $m(\xi, i y)$ в следующем виде:

$$
m(\xi, i y)=\sum_{l=-5}^{5} m^{(l)}(\xi, i y)
$$

где

$$
m^{(l)}(\xi, i y)=\sum_{k \equiv l(\bmod 11)}\left\|m_{2^{k}}\right\|_{\infty}^{\frac{\gamma-i y}{1-\gamma}} \psi\left(2^{-k} \xi\right)\left(J_{\lambda_{k}}^{(i y-\gamma) s_{0}} m_{2^{k}}\right)\left(2^{-k} \xi\right)
$$

и проверим, что

$$
\left\|m^{(l)}(\cdot, i y)\right\|_{\mathscr{M}\left(H_{p_{0}}\right)} \leqslant C(1+|y|)^{n+1} A^{\frac{1}{1-\gamma}}, \quad l=-5, \ldots, 5 .
$$

Фиксируя $j \in \mathbb{Z}$ и $l \in[-5,5]$, получаем

$$
m_{2^{j}}^{(l)}(\xi, i y)=\left\|m_{2^{k}}\right\|_{\infty}^{\frac{\gamma-i y}{1-\gamma}} \psi\left(2^{-l} \xi\right) \eta(\xi)\left(J_{\lambda_{k}}^{(i y-\gamma) s_{0}} m_{2^{k}}\right)\left(2^{-l} \xi\right), \quad k=j+l .
$$

Отсюда, используя [19, теорема 6.3.2], лемму 1, теорему о поточечных мультипликаторах (см., например, [22, с. 204]) и (36), последовательно находим

$$
\begin{aligned}
S_{j}^{(l)}: & =\left\|m_{2^{j}}^{(l)}(\cdot, i y)\right\|_{\infty}^{1-\theta_{0}}\left\|m_{2^{j}}^{(l)}(\cdot, i y)\right\|_{B_{\infty}^{s_{0}, \infty}}^{\theta_{0}} \\
& \leqslant C\left\|m_{2^{k}}\right\|_{\infty}^{\frac{\gamma}{1-\gamma}}\left\|J_{\lambda_{k}}^{(i y-\gamma) s_{0}} m_{2^{k}}\right\|_{\infty}^{1-\theta_{0}}\left\|\psi \eta\left(2^{l} \cdot\right) J_{\lambda_{k}}^{(i y-\gamma) s_{0}} m_{2^{k}}\right\|_{B_{\infty}^{s_{0}, \infty}}^{\theta_{0}} \\
& \leqslant C\left\|m_{2^{k}}\right\|_{\infty}^{\frac{\gamma}{1-\gamma}}\left\|J^{(i y-\gamma) s_{0}} m_{2^{k}}\right\|_{\infty}^{1-\theta_{0}}\left\|J_{\lambda_{k}}^{(i y-\gamma) s_{0}} m_{2^{k}}\right\|_{B_{\infty}^{s_{0}}, \infty}^{\theta_{0}} \\
& \leqslant C(1+|y|)^{n+1}\left\|m_{2^{k}}\right\|_{\infty}^{\frac{\gamma}{1-\gamma}+1-\theta_{0}}\left\|J_{\lambda_{k}}^{-\gamma s_{0}} m_{2^{k}}\right\|_{B_{\infty}^{s_{0}}, \infty}^{\theta_{0}},
\end{aligned}
$$

где $\theta_{0}=n\left(1 / p_{0}-1 / 2\right) / s_{0}$ и $k=j+l$.

Рассмотрим последний множитель в последнем неравенстве из (40). Используя лемму 4, а также (36), получаем

$$
\begin{aligned}
\left\|J_{\lambda_{k}}^{-\gamma s_{0}} m_{2^{k}}\right\|_{B_{\infty}^{s_{0}}, \infty} & \leqslant C\left\{\left\|J^{-\gamma s_{0}} \tau_{\lambda_{k}} m_{2^{k}}\right\|_{\infty}+\left\|J^{-\gamma s_{0}} \tau_{\lambda_{k}} m_{2^{k}}\left(\lambda_{k}^{-1} \cdot\right)\right\|_{\dot{B}_{\infty, \infty}^{s_{0}}}\right\} \\
& \leqslant C\left\{\left\|m_{2^{k}}\right\|_{\infty}+\lambda_{k}^{-s_{0}}\left\|J^{-\gamma s_{0}} \tau_{\lambda_{k}} m_{2^{k}}\right\|_{\dot{B}_{\infty}^{s_{0}}, \infty}\right\} .
\end{aligned}
$$

Заметим, что функция

$$
\mu(\xi)=\frac{|\xi|^{s_{0}-s}}{\left(1+|\xi|^{2}\right)^{\frac{\gamma s_{0}}{2}}}
$$

является мультипликатором Фурье в пространстве $\dot{B}_{\infty, \infty}^{s_{0}}$ (см., например, [22, c. 342]). Используя этот факт, свойство лифтинга для оператора $I^{s-s_{0}}$ (см., например, [22, с. 343]) и лемму 4 , находим

$$
\begin{aligned}
\left\|J^{-\gamma s_{0}} \tau_{\lambda_{k}} m_{2^{k}}\right\|_{\dot{B}_{\infty, \infty}^{s_{0}}} & \leqslant C\left\|I^{s-s_{0}} \tau_{\lambda_{k}} m_{2^{k}}\right\|_{\dot{B}_{\infty, \infty}^{s_{0}}} \\
& \leqslant C\left\|\tau_{\lambda_{k}} m_{2^{k}}\right\|_{\dot{B}_{\infty, \infty}^{s}} \leqslant C \lambda_{k}^{s}\left\|m_{2^{k}}\right\|_{\dot{B}_{\infty, \infty}^{s}} .
\end{aligned}
$$


Таким образом, объединяя (40)-(42) и учитывая при этом непрерывное вложение $B_{\infty, \infty}^{s} \subset L_{\infty}$ (см. лемму 3 ), имеем

$$
S_{j}^{(l)} \leqslant(1+|y|)^{n+1}\left\|m_{2^{j+l}}\right\|_{\infty}^{\frac{\gamma}{1-\gamma}+1-\theta_{0} \frac{s_{0}}{s}}\left\|m_{2^{j+l}}\right\|_{B_{\infty, \infty}^{s} \frac{\theta_{0}}{s}}^{s_{0}} .
$$

Остается воспользоваться неравенством (13), применяя которое к (43), мы сразу получаем (39) и, следовательно, (35). Завершается доказательство с помощью указанной выше интерполяционной теоремы.

ДокАЗАТЕЛЬСтво теоремы 5. Хорошо известно (см., например, [10], [4] или $\left[23\right.$, с. 119]), что если $f \in C_{0} \cap \dot{B}_{2,1}^{n / 2}$, то $f \in W_{0}\left(\mathbb{R}^{n}\right)$ и

$$
\|f\|_{W_{0}} \leqslant C\|f\|_{\dot{B}_{2,1}^{n / 2}}
$$

где $C$ - константа, не зависящая от $f$.

Таким образом, для того чтобы доказать принадлежность функции $f$ классу $W_{0}\left(\mathbb{R}^{n}\right)$, достаточно проверить, что

$$
\sum_{k=-\infty}^{+\infty} 2^{\frac{n}{2} k}\left\|\varphi_{k} * f\right\|_{2}=\sum_{k=0}^{+\infty}+\sum_{k=-\infty}^{-1}=S_{1}+S_{2}<\infty .
$$

1) Рассмотрим сначала случай $0<q, r \leqslant 2$. Пусть $s_{1}=s-n(1 / r-1 / 2)$. Используя вложение $\dot{B}_{r, \infty}^{s} \subset \dot{B}_{2, \infty}^{s_{1}}$ (см. лемму 2 , (ii)), находим

$$
S_{1} \leqslant \sup _{j \in \mathbb{N} \cup\{0\}} 2^{s_{1} j}\left\|\varphi_{j} * f\right\|_{2} \sum_{k=0}^{\infty} 2^{\left(\frac{n}{2}-s_{1}\right) k} \leqslant\|f\|_{\dot{B}_{2, \infty}^{s_{1}}} \sum_{k=0}^{\infty} 2^{\left(\frac{n}{r}-s\right) k} \leqslant C\|f\|_{\dot{B}_{r, \infty}^{s}} .
$$

Аналогично, используя вложение $\dot{B}_{q, \infty}^{\sigma} \subset \dot{B}_{2, \infty}^{\sigma_{1}}$, где $\sigma_{1}=\sigma-n(1 / q-1 / 2)$, получаем

$$
S_{2} \leqslant C\|f\|_{\dot{B}_{q, \infty}^{\sigma}} .
$$

Объединяя (45) и (46), имеем

$$
\|f\|_{W_{0}} \leqslant C\left\{\|f\|_{\dot{B}_{q, \infty}^{\sigma}}+\|f\|_{\dot{B}_{r, \infty}^{s}}\right\} .
$$

Чтобы получить неравенство (19), мы применяем к (47) стандартный переход к мультипликативным неравенствам с помощью функции $f_{\varepsilon}(x)=f(\varepsilon x)$ и леммы 4 (см., например, [24, с. 311]).

2) Рассмотрим теперь случай $0<q<2<r \leqslant \infty$. Сумма $S_{2}$ оценивается так же, как в (46).

Оценим сумму $S_{1}$. Обозначим $\lambda=\frac{r(2-q)}{2(r-q)}$. Применяя лемму 6 при $\alpha=q$ и $\beta=r$, находим

$$
\begin{aligned}
S_{1} & \leqslant \sum_{k=0}^{\infty} 2^{\frac{n}{2} k}\left\|\varphi_{k} * f\right\|_{q}^{1-\lambda}\left\|\varphi_{k} * f\right\|_{r}^{\lambda} \\
& \leqslant\left(\sup _{j \in \mathbb{Z}} 2^{\sigma j}\left\|\varphi_{j} * f\right\|_{q}\right)^{1-\lambda}\left(\sup _{j \in \mathbb{Z}} 2^{s j}\left\|\varphi_{j} * f\right\|_{r}\right)^{\lambda} \sum_{k=0}^{\infty} 2^{\left(\frac{n}{2}-(1-\lambda) \sigma-\lambda s\right) k} \\
& =\|f\|_{\dot{B}_{q, \infty}^{\sigma}}^{1-\lambda}\|f\|_{\dot{B}_{r, \infty}^{s}}^{\lambda} \sum_{k=0}^{\infty} 2^{\frac{q r(s-\sigma)}{r-q}\left(\frac{1}{2}-(1-\theta) \frac{1}{q}-\theta \frac{1}{r}\right) k} \leqslant C\|f\|_{\dot{B}_{q, \infty}^{\sigma}}^{1-\lambda}\|f\|_{\dot{B}_{r, \infty}^{s}}^{\lambda} .
\end{aligned}
$$


Таким образом, из (44), объединяя (46) и (48), имеем

$$
\|f\|_{W_{0}} \leqslant C\left\{\|f\|_{\dot{B}_{q, \infty}^{\sigma}}+\|f\|_{\dot{B}_{q, \infty}^{\sigma}}^{1-\lambda}\|f\|_{\dot{B}_{r, \infty}^{s}}^{\lambda}\right\} .
$$

Остается только сделать переход к мультипликативному неравенству.

3) Случай $2<q \leqslant \infty, 0<r<2$ доказывается аналогично.

ДокАЗАТЕЛЬСТво ПРЕДЛОЖЕНИЯ 1. В качестве функции, удовлетворяющей условиям предложения, возьмем $m=m_{\alpha, \beta}$ из (21), где числа $\alpha$ и $\beta$ мы выбираем так, чтобы $\beta \geqslant s \theta^{*} \alpha$, но $\beta<\alpha n(1 / p-1 / 2)$. В этом случае в силу леммы 5 , (i) имеем $m \notin \mathscr{M}\left(H_{p}\right)$. Таким образом, для доказательства предложения достаточно проверить, что для функции $m$ выполнено (14).

Проводя простые вычисления, получаем

$$
\left\|m_{2^{j}}\right\|_{q} \leqslant C \cdot 2^{-\left(\beta+\frac{n}{q}\right) j} \leqslant C \cdot 2^{-\beta j} .
$$

Далее, используя мультипликативное неравенство (см., например, [23, с. 111])

$$
\|f\|_{B_{r, \infty}^{\lambda k}} \leqslant C\|f\|_{B_{r, \infty}^{0}}^{1-\lambda}\|f\|_{B_{r, \infty}^{k}}^{\lambda}, \quad 0<\lambda<1
$$

где $C$ - положительная константа, не зависящая от функции $f$, и лемму 3 , находим

$$
\left\|m_{2^{j}}\right\|_{B_{r, \infty}^{s}} \leqslant C\left\|m_{2^{j}}\right\|_{r}^{1-\frac{s}{k}}\left\|m_{2^{j}}\right\|_{W_{r}^{k}}^{\frac{s}{k}} \leqslant C \cdot 2^{-\left(\beta+\frac{n}{r}-s \alpha\right) j} \leqslant C \cdot 2^{-(\beta-s \alpha) j} .
$$

Таким образом, объединяя (49) и (51), имеем

$$
\left\|m_{2^{j}}\right\|_{L_{q}}^{1-\theta^{*}}\left\|m_{2^{j}}\right\|_{B_{r, \infty}^{s}}^{\theta^{*}} \leqslant C \cdot 2^{-\left(\beta-\theta^{*} s \alpha\right) j} \leqslant C,
$$

откуда сразу следует (14). Предложение доказано.

ДоКАЗАТЕЛЬСТво ПРЕДЛОЖЕНИЯ 2. 1) Рассмотрим случай $0<p \leqslant 1$.

Пусть $\psi \in \mathscr{S}\left(\mathbb{R}^{n}\right), \operatorname{supp} \psi \in\{|\xi| \leqslant 1\}$ и $\psi(\xi)=1$ при $|\xi| \leqslant 1 / 2$. Положим

$$
m(\xi)=\sum_{j=N_{\varepsilon}}^{\infty} 2^{-n\left(\frac{1}{p}-1\right) k_{j}} \psi\left(2^{k_{j}}\left(\xi-2^{\varepsilon k_{j}} e_{1}\right)\right),
$$

где $e_{1}=(1,0, \ldots, 0) \in \mathbb{R}^{n},\left\{k_{j}\right\} \subset \mathbb{N}$ - произвольная лакунарная последовательность такая, что $\inf _{j} \frac{k_{j+1}}{k_{j}} \geqslant 10$, а $N_{\varepsilon}$ выбрано так, чтобы существовало единственное $j$ такое, что при некотором заданном $\varepsilon>0$ и любом $\delta>0$

$$
\left\{\frac{\delta}{4} \leqslant|\xi| \leqslant 4 \delta\right\} \cap\left\{\left|\xi-2^{\varepsilon k_{j}}\right| \leqslant 2^{-k_{j}}\right\} \neq \varnothing .
$$

Тогда очевидно, что

$$
m(\delta \xi) \eta(\xi)=2^{-n\left(\frac{1}{p}-1\right) k_{j}} \psi\left(2^{k_{j}} \delta \xi-2^{(\varepsilon+1) k_{j}} e_{1}\right) \eta(\xi),
$$

где $j$ такое, что $2^{\varepsilon k_{j}} \asymp \delta$. 
Используя неравенство (50) и лемму 3 , нетрудно проверить, что

$$
\left\|m_{\delta}\right\|_{q}^{1-\theta}\left\|m_{\delta}\right\|_{B_{r, \infty}^{s}}^{\theta} \leqslant C\left(2^{\left((1+\varepsilon)\left(s \theta-(1-\theta) \frac{n}{q}-\theta \frac{n}{r}\right)-n\left(\frac{1}{p}-1\right)\right) k_{j}}+1\right),
$$

где $C$ - положительная константа, не зависящая от $\delta$ и $k_{j}$. Если

$$
(1+\varepsilon)\left(\frac{1-\theta}{q}+\frac{\theta}{r}\right) \geqslant \frac{1}{2}+\varepsilon\left(\frac{1}{p}-\frac{1}{2}\right),
$$

то правая часть неравенства (52) будет конечна. Таким образом, при соответствующем выборе параметра $\varepsilon$, существование которого гарантируется неравенствами (15) и (16), мы получим (17).

Покажем, что

$$
m \notin \mathscr{M}\left(H_{p}\right) .
$$

Для этого воспользуемся схемой доказательства теоремы 5, b из [9].

Введем функции

$$
\begin{gathered}
g(\xi)=\sum_{j=N_{\varepsilon}}^{\infty} j^{-\frac{1}{p}} \psi\left(\xi-2^{\varepsilon k_{j}} \xi\right) \\
f(x)=\mathscr{F}^{-1}(m g)(x)=\sum_{j=N_{\varepsilon}}^{\infty} j^{-\frac{1}{p}} 2^{-\frac{n k_{j}}{p}} \mathscr{F}^{-1} \psi\left(\frac{x}{2^{k_{j}}}\right) e^{i \cdot 2^{\varepsilon k_{j}} x_{1}} .
\end{gathered}
$$

Заметим, что $\mathscr{F}^{-1} g \in H_{p}\left(\mathbb{R}^{n}\right), 0<p \leqslant 1$. Этот факт легко следует из работы $\left[9\right.$, с. 14]. Таким образом, показав, что $f \notin H_{p}\left(\mathbb{R}^{n}\right), 0<p \leqslant 1$, мы получим (53). Достаточно проверить, что $f \notin L_{p}\left(\mathbb{R}^{n}\right)$ (см. [8, теорема 3.4]).

Выбирая $l$ достаточно большим, представим функцию $f$ в следующем виде:

$$
f(x)=\sum_{j<l}+\sum_{j=l}+\sum_{j>l}=f_{1}(x)+f_{2}(x)+f_{3}(x) .
$$

Учитывая, что $\mathscr{F}^{-1} \psi \in \mathscr{S}\left(\mathbb{R}^{n}\right)$, получаем

$$
\int_{2^{k_{l}<<x \mid \leqslant 2^{k_{l}+1}}}\left|f_{2}(x)\right|^{p} d x=\frac{1}{l} \int_{1<|x| \leqslant 2}\left|\mathscr{F}^{-1} \psi(x)\right|^{p}=\frac{C}{l} .
$$

Далее, поскольку

$$
\left|f_{3}(x)\right| \leqslant C \sum_{j>l} j^{-\frac{1}{p}} 2^{-\frac{n k_{j}}{p}} \leqslant C l^{-\frac{1}{p}} 2^{-\frac{n k_{l+1}}{p}}
$$

имеем

$$
\int_{2^{k_{l}}<|x| \leqslant 2^{k_{l}+1}}\left|f_{3}(x)\right|^{p} d x \leqslant \frac{C}{l \cdot 2^{n\left(k_{l+1}-k_{l}\right)}} .
$$

Рассмотрим $f_{1}$. Пусть $r>n / p$ и $2^{k_{l}} \leqslant|x| \leqslant 2^{k_{l}+1}$. Поскольку $\left|\mathscr{F}^{-1} \psi(x)\right| \leqslant$ $C|x|^{-r}$ при $|x|>1$, то

$$
\left|f_{1}(x)\right| \leqslant C \sum_{j<l} j^{-\frac{1}{p}} 2^{-\frac{n k_{j}}{p}+r\left(k_{j}-k_{l}\right)} \leqslant C l^{-\frac{1}{p}} 2^{-\frac{n k_{l-1}}{p}+r\left(k_{l-1}-k_{l}\right)}
$$


и, следовательно,

$$
\int_{2^{k_{l}<|x| \leqslant 2^{k_{l}+1}}}\left|f_{1}(x)\right|^{p} d x \leqslant \frac{C}{l \cdot 2^{(r p-n)\left(k_{l}-k_{l-1}\right)}} .
$$

Таким образом, из (55) и (56), учитывая, что $k_{l+1}-k_{l} \rightarrow \infty$ при $l \rightarrow \infty$, имеем

$$
\int_{2^{k_{l}<<x \mid \leqslant 2^{k_{l}+1}}}\left|f_{1}(x)\right|^{p}+\left|f_{3}(x)\right|^{p} d x=o\left(\frac{1}{l}\right), \quad l \rightarrow \infty .
$$

Объединяя (55) и (57), получаем, что для достаточно больших $l$

$$
\int_{2^{k_{l}<|x| \leqslant 2^{k_{l}+1}}}|f(x)|^{p} d x \geqslant \frac{C}{l} .
$$

Из последнего неравенства сразу следует, что $f \notin L_{p}\left(\mathbb{R}^{n}\right)$.

2) Теперь рассмотрим случай $1<p<2$. Положим

$$
m(\xi)=\sum_{j=1}^{\infty} e^{i \cdot 2^{j} x_{1}} \psi\left(\xi-2^{j} e_{1}\right) .
$$

Известно (см. [22, с. 182]), что

$$
m \notin \bigcup_{1<p<\infty, p \neq 2} \mathscr{M}\left(L_{p}\right) .
$$

Выполняя простые вычисления, используя при этом (50) и лемму 3 , находим, что

$$
\left\|m_{\delta}\right\|_{q}^{1-\theta}\left\|m_{\delta}\right\|_{B_{r, \infty}^{s}}^{\theta} \leqslant C\left(\delta^{\left(s \theta-(1-\theta) \frac{n}{q}-\theta \frac{n}{r}\right)}+1\right),
$$

где $C$ - положительная константа, не зависящая от $\delta$. Таким образом, поскольку $m_{\delta} \equiv 0$ при $\delta<1 / 4$, для $m$ выполняется неравенство (17) при условии (16).

ДОКАЗАТЕЛЬСТво ПРЕДЛОЖЕНИЯ 3. По аналогии с доказательством предложения 1 рассмотрим функцию $f=m_{\alpha, \beta}$ (см. $\left.(21)\right)$.

Используя вложения из леммы 3 , нетрудно проверить, что $f \in \dot{B}_{q, \infty}^{\sigma} \cap \dot{B}_{r, \infty}^{s}$, если

$$
\beta-\sigma(\alpha-1)>\frac{n}{q}, \quad \beta-s(\alpha-1)>\frac{n}{r} .
$$

Пусть $\varepsilon^{\prime}$ и $\varepsilon^{\prime \prime}$ - некоторые достаточно малые положительные числа. Положим

$$
\alpha=\frac{s-\frac{n}{r}+\frac{n}{q}-\sigma+\varepsilon^{\prime}-\varepsilon^{\prime \prime}}{s-\sigma}, \quad \beta=\frac{n\left(\frac{s}{q}-\frac{\sigma}{r}\right)+\varepsilon^{\prime} s-\varepsilon^{\prime \prime} s}{s-\sigma} .
$$

Варьируя соответствующим образом $\varepsilon^{\prime}$ и $\varepsilon^{\prime \prime}$, можно добиться того, чтобы выполнялись неравенства (58) и $\alpha, \beta>0, \alpha \neq 1$.

Далее, из (20) и (58) имеем

$$
\frac{\alpha n}{2}>\beta-\left((1-\theta) \varepsilon^{\prime}+\theta \varepsilon^{\prime \prime}\right)
$$

Таким образом, мы можем выбрать $\alpha$ и $\beta$ так, чтобы $\frac{\alpha n}{2}>\beta$. В этом случае в силу леммы 5, (ii) имеем $f \notin W_{0}\left(\mathbb{R}^{n}\right)$. Предложение доказано. 


\section{Список литературы}

1. С. Г. Михлин, "О мультипликаторах интегралов Фурье”, Докл. АН СССР, 109:4 (1956), 701-703.

2. L. Hörmander, "Estimates for translation invariant operators in $L^{p}$ spaces", Acta Math., 104:1-2 (1960), 93-140.

3. W. Littman, "Multipliers in $L^{p}$ and interpolation", Bull. Amer. Math. Soc., 71 (1965), 764-766.

4. J. Peetre, "Applications de la théorie des espaces d'interpolation dans l'analyse harmonique", Ricerche Mat., 15 (1966), 3-36.

5. Ch. Fefferman, E. M. Stein, " $H^{p}$ spaces of several variables", Acta Math., 129:3-4 (1972), 137-193.

6. A. P. Calderón, A. Torchinsky, "Parabolic maximal functions associated with a distribution. II", Advances in Math., 24:2 (1977), 101-171.

7. A. Miyachi, "On some Fourier multipliers for $H^{p}\left(\mathbb{R}^{n}\right)$ ", J. Fac. Sci. Univ. Tokyo Sect. IA Math., 27:1 (1980), 157-179.

8. A. Miyachi, "On some singular Fourier multipliers", J. Fac. Sci. Univ. Tokyo Sect. IA Math., 28:2 (1981), 267-315.

9. A. Baernstein II, E. T. Sawyer, "Embedding and multiplier theorems for $H^{p}\left(\mathbb{R}^{n}\right)$ ", Mem. Amer. Math. Soc., 53, № 318, Amer. Math. Soc, Providence, RI, 1985.

10. О.В. Бесов, "К теореме Хермандера о мультипликаторах Фурье", Исследования по теории дифференцируемых функций многих переменных и ее приложениям. ч. 11, Тр. МИАН СССР, 173, Наука, М., 1986, 3-13; англ. пер.: О. V. Besov, "On Hörmander's theorem on Fourier multipliers", Proc. Steklov Inst. Math., 173 (1987), 1-12.

11. П. И. Лизоркин, "Предельные случаи теорем о $\mathscr{F} L_{p}$-мультипликаторах", Исследования по теории дифференцируемых функций многих переменных и ее приложениям. ч. 11, Тр. МИАН СССР, 173, Наука, М., 1986, 164-180; англ. пер.: P. I. Lizorkin, "Limiting cases of theorems on $\mathscr{F} L_{p}$-multipliers", Proc. Steklov Inst. Math., 173 (1987), 1-12.

12. C.W. Onneweer, T.S. Quek, "On $H^{p}\left(\mathbb{R}^{n}\right)$-multipliers of mixed-norm type", Proc. Amer. Math. Soc., 121:2 (1994), 543-552.

13. Р. М. Тригуб, "Мультипликаторы в пространствах Харди $H_{p}\left(D^{m}\right)$ при $p \in(0,1]$ и аппроксимативные свойства методов суммирования степенных рядов", Матем. сб., 188:4 (1997), 145-160; англ. пер.: R. M. Trigub, "Multipliers in the Hardy spaces $H_{p}\left(D^{m}\right)$ with $p \in(0,1]$ and approximation properties of summability methods for power series", Sb. Math., 188:4 (1997), 621-638.

14. Ю.С. Коломойцев, "Обобщение одного достаточного условия для мультипликаторов Фурье", Укр. матем. журн., 64:10 (2012), 1373-1380; англ. пер.: Yu. S. Kolomoitsev, "Generalization of one sufficient condition for Fourier multipliers", Ukrainian Math. J., 64:10 (2013), 1562-1571.

15. E. Liflyand, R. Trigub, "Conditions for the absolute convergence of Fourier integrals", J. Approx. Theory, 163:4 (2011), 438-459.

16. E. Liflyand, "On absolute convergence of Fourier integrals", Real Anal. Exchange, 36:2 (2010), 353-360.

17. E. Liflyand, S. Samko, R. Trigub, "The Wiener algebra of absolutely convergent Fourier integrals: an overview", Anal. Math. Phys., 2:1 (2012), 1-68.

18. И. М. Стейн, Сингулярные интеграль и дифференииальные свойства функиий, Мир, М., 1973; пер. с англ.: Е. M. Stein, Singular integrals and differentiability properties of functions, Princeton Math. Ser., 30, Princeton Univ. Press, Princeton, NJ, 1970. 
19. Й. Берг, Й. Лефстрем, Интерполяционные пространства. Введение, Мир, М., 1980; пер. с англ.: J. Bergh, J. Löfström, Interpolation spaces. An introduction, Springer-Verlag, Berlin-Heidelberg-New York, 1976.

20. A. Beurling, "Sur les intégrales de Fourier absolument convergentes et leur application à une transformation fonctionnelle", Proc. IX Congrès de Math. Scand., Helsingfors, 1938, 345-366.

21. J. Löfström, "Besov spaces in theory of approximation", Ann. Mat. Pura Appl. (4), 85 (1970), 93-184.

22. Х. Трибель, Теория функииональных пространств, Мир, М., 1986; пер. с англ.: Н. Triebel, Theory of function spaces, Monogr. Math., 78, Birkhaüser, Basel-Boston-Stuttgart, 1983.

23. J. Peetre, New thoughts on Besov spaces, Duke Univ. Press, Durham, NC, 1976.

24. С. М. Никольский, Приближение функций многих переменных и теоремы вложения, 2-е изд., Наука, М., 1977; англ. пер. 1-го изд.: S. M. Nikolśkiǐ, Approximation of functions of several variables and imbedding theorems, Springer-Verlag, New York-Heidelberg, 1975.

25. О.В.Бесов, В. П. Ильин, С. М. Никольский, Интегральные представления функиий и теоремы вложения, Наука, M., 1975; англ. пер.: О. V. Besov, V. P. Il'in, S. M. Nikol'skiu, Integral representations of functions and imbedding theorems, v. I, II, Winston, Washington, DC; Wiley, New York-Toronto, ON-London, 1979.

26. S. Wainger, "Special trigonometric series in k-dimensions", Mem. Amer. Math. Soc., 59, Amer. Math. Soc, Providence, RI, 1965.

27. И. Стейн, Г. Вейс, Введение в гармонический анализ на евклидовых пространствах, Мир, М., 1974; пер. с англ.: Е. M. Stein, G. Weiss, Introduction to Fourier analysis on Euclidean spaces, Princeton Math. Ser., 32, Princeton Univ. Press, Princeton, NJ, 1971.

Юрий СЕРГЕЕВич КоЛОМОйцев

(Yuriy S. Kolomoitsev)

Поступило в редакцию

Институт прикладной математики и механики

30.11 .2012

НАН Украины, г. Донецк

E-mail: kolomus1@mail.ru 